\title{
In vivo Mouse Mammary Gland Formation
}

Heng Sun, Xin Zhang, Un In Chan, Sek Man Su, Sen Guo, Xiaoling Xu and Chuxia Deng*

Faculty of Health Sciences, University of Macau, Macau SAR, China

*For correspondence: cxdeng@um.edu.mo

\begin{abstract}
[Abstract] For years, the mammary gland serves as a perfect example to study the self-renew and differentiation of adult stem cells, and the regulatory mechanisms of these processes as well. To assess the function of given genes and/or other factors on stemness of mammary cells, several in vitro assays were developed, such as mammospheres formation assay, detection of stem cell markers by mRNA expression or flow cytometry and so on. However, the capacity of reconstruction of whole mount in the cleared fat pad of recipient female mice is a golden standard to estimate the stemness of the cells. Here we described a step-by-step protocol for in vivo mammary gland formation assay, including preparation of "cleared" recipients and mammary cells for implantation, the surgery process and how to assess the experimental results. Combined with manipulation of mammary cells via gene editing and /or drug treatment, this protocol could be very useful in the researches of mammary stem cells and mammary development.
\end{abstract}

Keywords: Mammary gland, Whole mount, Mammary stem cells, Tissue reconstruction, Cell transplantation

[Background] As one of the most typical organ of mammals, the mammary gland (MG) is an exocrine gland and responsible for lactation. The development of $M G$ is under the control of certain sexual hormones, whose levels precisely regulate the structure, cellular composition and functional changes of MG in different developmental stages (Hennighausen and Robinson, 2005). Many genetic and environmental factors are involved in the regulation of mammary stem cells and the MG development. To study the functions and mechanisms of these factors, several methods have been developed, especially for the assessment of stemness of mammary cells. Previous studies demonstrated that only the basal cells instead of luminal cells of MG are able to reconstruct the epithelial tree in the cleared fat pads of recipient female mice, indicating the mammary stem cells only exist in the basal lineage (Van Keymeulen et al., 2011). Later, many studies including ours, uncovered several markers for mammary stem cells (Prater et al., 2014; Wang et al., 2015; Sun et al., 2018), whose function in stemness maintenance was confirmed by the in vivo MG formation assay. In this protocol, we described in detail how to prepare the donor mammary cells, how to remove the endogenous epithelia from the fad pads of recipients female mice, how to perform the implantation, as well as how to assess the experimental results. Besides of verifying mammary stem cell markers, this protocol could be also very useful in other researches regarding mammary stem cells and mammary development when combined with manipulation of mammary cells via gene editing, gene overexpression or knockdown, and/or drug treatment. 


\section{Materials and Reagents}

1. $50 \mathrm{ml}$ centrifuge tubes (Corning, catalog number: CLS430829)

2. $1.5 \mathrm{ml}$ micro-tubes (Axygen, catalog number: MCT150-C-S)

3. Pipette tips $10 \mu \mathrm{l}, 200 \mu \mathrm{l}$ and 1,000 $\mu \mathrm{l}$ (Axygen, catalog numbers: T-400, T-200-Y and T-1000B)

4. FACS tubes (FALCON, catalog number: 352058)

5. $40 \mu \mathrm{m}$ cell strainer (FALCON, catalog number: 352340 )

6. Cell counting chambers (Nexcelom Bioscience, catalog number: CHT4-SD100)

7. $0.2 \mu \mathrm{m}$ Nalgene syringe filter (Thermo Fisher Scientific, catalog number: 725-2520)

8. $50 \mathrm{ml}$ syringe (TERUMO, catalog number: SS-50LE)

9. $1 \mathrm{ml}$ syringe without needle (TERUMO, catalog number: SS-01T)

10. $27 \mathrm{G} \times 1 / 2$ " needle (TERUMO, catalog number: NN-2713R)

11. Microscope slides (Thermo Fisher Scientific, catalog number: J1801ASH)

12. Cover glass (Thermo Fisher Scientific, catalog numbers: 102222 and 102240)

13. Three-month-old female FVB mice for preparation of donor cells (as donors, provided by Animal facility core, Faculty of Health Sciences, University of Macau)

14. Three-week-old virgin female nude mice (as recipients, provided by Animal facility core, Faculty of Health Sciences, University of Macau)

15. DMEM/F12 medium (Thermo Fisher Scientific, catalog number: 11330032)

16. Fetal Bovine Serum (FBS) (Thermo Fisher Scientific, catalog number: 26140079)

17. HBSS (Thermo Fisher Scientific, catalog number: 14140112)

18. Hydrocortisone (Sigma-Aldrich, catalog number: H0888)

19. Insulin (Sigma-Aldrich, catalog number: $91077 \mathrm{C}$ )

20. EGF (Thermo Fisher Scientific, catalog number: PHG0313)

21. Cholera toxin (Sigma-Aldrich, catalog number: C8052)

22. Collagenase Type 3 (Worthington Biochemical Corporation, catalog number: LS004183)

23. Hyaluronidase (Sigma-Aldrich, catalog number: H3506)

24. Dispase II (Roche, catalog number: 04942078001)

25. Deoxyribonuclease I (Worthington Biochemical Corporation, catalog number: LS002145)

26. HEPES (1 M) (Thermo Fisher Scientific, catalog number: 15630080)

27. DPBS (Thermo Fisher Scientific, catalog number: 14190250)

28. $0.25 \%$ Trypsin-EDTA (Thermo Fisher Scientific, catalog number: 25200056)

29. 1x RBC lysis buffer (Thermo Fisher Scientific, catalog number: 00-4333-57)

30. EasySeq Mouse Epithelial Cell Enrichment kit (STEMCELL Technologies, catalog number: 19868)

31. Trypan blue (STEMCELL Technologies, catalog number: 07050)

32. Anti-mouse CD24-PE-Cy7 antibody (BD Biosciences, catalog number:560536)

33. Anti-mouse CD29-APC antibody (Biolegend, catalog number:102216) 
34. DAPI solution (1 $\mathrm{mg} / \mathrm{ml}$ ) (Thermo Fisher Scientific, catalog number: 62248 )

35. Albumin, Bovine (BSA) (VWR LIFE SCIENCE, catalog number: VWRV0332)

36. EDTA (0.5 M), pH 8.0 (Thermo Fisher Scientific, catalog number: AM9260G)

37. DPBS (Thermo Fisher Scientific, catalog number: 14190250)

38. PBS Tablets (Thermo Fisher Scientific, catalog number: 18912014)

39. 2,2,2-Tribromoethanol (Sigma-Aldrich, catalog number: T48402)

40. 2-Methyl-2-butanol (Sigma-Aldrich, catalog number: 152463)

41. Ethanol (Sigma-Aldrich, catalog number: E7023)

42. Chloroform (Sigma-Aldrich, catalog number: C2432)

43. Acetic acid (Sigma-Aldrich, catalog number: A6283)

44. Carmine (Sigma-Aldrich, catalog number: C1022)

45. Aluminum potassium sulfate (Sigma-Aldrich, catalog number: A7210)

46. Xylenes (Sigma-Aldrich, catalog number: 214736)

47. DPX Mountant (Sigma-Aldrich, catalog number: O6522)

48. Implantation buffer (see Recipes)

49. Anesthetics (Avertin, see Recipes)

50. Carnoy's fix buffer (see Recipes)

51. Carmine-alum staining solution (see Recipes)

\section{Equipment}

1. Sterile scissors and forceps

2. Tapes (to restrain the mice)

3. Wound closures applier (RWD, catalog number: 12020-09)

4. $9 \mathrm{~mm}$ wound clips (RWD, catalog number: 12022-09)

5. Warming pad (Lab Animal Technology Develop Co., catalog number: LAT-BW2)

6. Pipette

7. Biosafety cabinet for cell culture work

8. Chemical hood

9. Centrifuge with adaptors for $50 \mathrm{ml}$ centrifuge tubes and FACS tubes, for use at room temperature.

10. EasySep Magnet (STEMCELL Technologies, catalog number: 18000)

11. Cell counter (Nexcelom Bioscience, model: Cellometer Auto 2000)

12. BD FACSAria III for cell sorting

13. Leica-M165FC stereo microscope

14. Magnetic stirrer (IKA, RH basic) 


\section{Software}

\section{BD FACSDiva 6.1 for BD FACSAria III}

2. ImageJ

\section{Procedure}

A. Prepare donor MG cells (details are descripted in Sun et al., 2020)

1. Sacrifice the donor female mice and remove the fourth inguinal MGs under clean and sterile condition.

2. Digest the MGs into single cells. In brief, the mammary glands were minced, washed in DPBS, and digested in DMEM/F-12, which contained 300 units $/ \mathrm{ml}$ collagenase III, 100 units $/ \mathrm{ml}$ hyaluronidase, $5 \%$ FBS , $5 \mathrm{\mu g} / \mathrm{ml}$ insulin, $10 \mathrm{ng} / \mathrm{ml} \mathrm{EGF,} 20 \mathrm{ng} / \mathrm{ml}$ Cholera toxin, and $500 \mathrm{ng} / \mathrm{ml}$ hydrocortisone, for about $1 \mathrm{~h}$ at $37{ }^{\circ} \mathrm{C}, 5 \% \mathrm{CO}_{2}$. The resultant organoid suspension was sequentially re-suspended in DMEM/F-12 supplemented with $5 \mathrm{mg} / \mathrm{ml}$ dispase $\mathrm{Il}$ and $0.1 \mathrm{mg} / \mathrm{ml}$ DNase I for $5 \mathrm{~min}$ at $37^{\circ} \mathrm{C}$, and then digested with $0.25 \%$ trypsin-EDTA for $2 \mathrm{~min}$, and treated with RBC lysis buffer for $3 \mathrm{~min}$ to remove the red blood cells before filtration through a $40-\mu \mathrm{m}$ cell strainer to obtain single-cell suspension.

3. Enrich the epithelial cells using an EasySeq Mouse Epithelial Cell Enrichment kit according to the manufacturer and stain the MG cells with anti-mouse CD24-PE-Cy7 antibody (1:200), antimouse CD29-APC antibody (1:200), and DAPI $(1 \mu \mathrm{g} / \mathrm{ml})$ in FACS buffer in ice for $30 \mathrm{~min}$.

4. Sort out the DAPI-CD24 ${ }^{\mathrm{Mid}} \mathrm{CD} 29^{\mathrm{Hi}}$ (basal cells) and DAPI-CD24 ${ }^{\mathrm{Hi}} \mathrm{CD} 29^{\mathrm{Lo}}$ (luminal cells) cells separately as donor cells.

5. Wash the cells with DPBS and re-suspend the cells at given concentrations ( $10 \mu \mathrm{l}$ of cells/ fat pad) with implantation buffer (Recipe 1).

B. Clear the fat pads of recipient female mice

1. Anesthetize a 3-week-old female nude mouse with Avertin (Recipe 2) (administered i.p., at a dose of $250 \mathrm{mg} / \mathrm{kg}$ body weight). Select mice those are $<12 \mathrm{~g}$ to ensure that mammary ductal growth has not been initiated.

2. Firmly restrain the mouse with abdomen up by adhering its limbs into a foam surface using tapes (Figure 1A).

3. Sterilize the abdomen with $70 \%$ alcohol.

4. Grasp the skin with forceps a few millimeters above the pelvis and lift it up from the abdomen. Make a parallel incision (about $10 \mathrm{~mm}$ ) along the mouse's abdomen without puncturing the peritoneum with scissors and forceps.

5. Then make a Y-type incision from the bottom of the midline incision to the hip region just above the legs. Pull the skin back from the peritoneum using forceps to expose the mammary gland (Figure 1B). 
6. Cut off the bridge between the fourth and fifth mammary glands to prevent the epithelium of the fifth gland from growing into the fourth cleared fat pads.

7. Divide the fat pad of the fourth mammary gland by cutting across it with scissors just near ventral (the side towards nipple) of the node. Then remove the portion of fat pad with the lymph node and the nipple, and leave the other part as cleared fat pad for MG cells implantation later (Figures 1B-1D).

8. Put the removed mammary piece on a microscope slide for fixing and staining with carminealum later to confirm that the entire rudimentary epithelial bud has been removed.

9. Repeat the Steps B6-B8, if desired, on the contralateral mammary gland.

C. Implant the donor MG cells into the cleared fat pads

1. After clearing the mammary fat pad, directly inject the $M G$ cells $(10 \mu l)$ prepared from Procedure A into the remaining part of the fat pad by using $1 \mathrm{ml}$ syringe with $27 \mathrm{G} \times 1 / 2$ " needle (Figures $1 \mathrm{E}$ and $1 \mathrm{~F})$.

2. Close the skin incisions with wound clips (Figure 1G).

3. Place the mouse on a warming pad until it regains consciousness. And keep monitoring the mouse for up to 1 week for any sign of infection or other surgical complications.
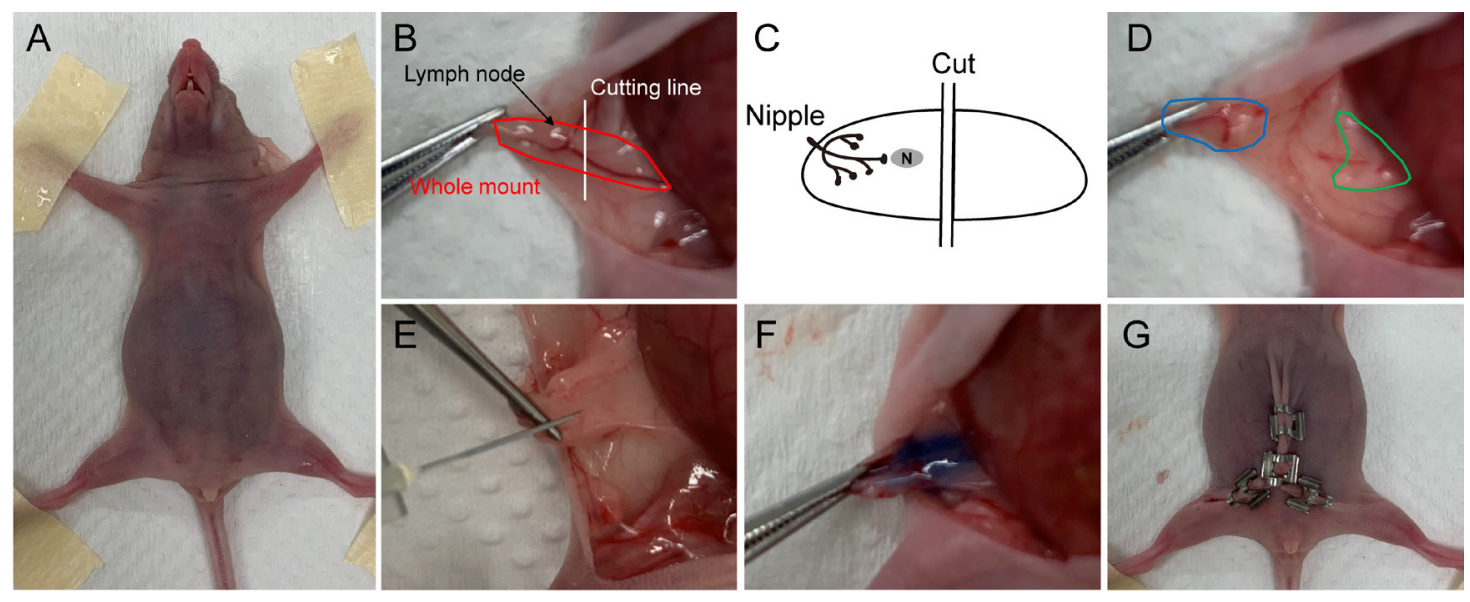

Figure 1. Surgical process. A. The anesthetized mouse was restrained on a foam surface. B. The fourth mammary gland was exposed (the locations of lymph node and cutting line were indicated). C. A schematic diagram shows the locations of nipple, lymph node (labeled as "N") and the cutting line. D. Divide the MG into two parts, the blue circled part was to be removed and the green circled part was the cleared fat pad. E. Injecte the donor cells into the cleared fat pad. F. Cleared fat pad was implanted with MG cells. G. Close the incisions with wound clips.

D. Remove, fix and stain the MG

1. About 8 to 10 weeks after implantation, sacrifice the recipient mouse, dissect out the fourth MG (similar operation as in Procedure B, just remove all the fat pad).

2. Transfer the entire MG on a microscope slide, and use blunt end forceps to spread out the MG 
over the glass slide as much as possible without damaging the tissue.

3. Keep the MG on the slide for $5 \mathrm{~min}$ at room temperature to allow the MG to stick to the glass surface.

4. Submerge the slide in a $50 \mathrm{ml}$ centrifuge tube containing Carnoy's fix buffer (Recipe 3), and fix the tissue overnight at room temperature.

5. Wash the slide with $70 \%$ ethanol for 30 min, then wash it with $50 \%, 30 \%$ ethanol and PBS for 10 min of each step.

6. Stain the MG with carmine-alum staining solution (Recipe 4) at $4{ }^{\circ} \mathrm{C}$ overnight.

Note: The staining can be continued for several days.

7. Dehydrate the MG by washing the slide with a gradient ethanol $(70 \%, 95 \%, 100 \%, 15$ min for each concentration).

8. Submerge the MG in xylene overnight at room temperature in the chemical hood; to remove the fat tissue.

9. Mount the slide with Permout in the chemical hood for long-term storage.

E. Assess the MG formation efficiency

1. Take pictures of the stained whole mounts by using a Leica-M165FC stereo microscope.

2. Measure the size of the reconstructed epithelia and whole mounts by analyzing the picture with ImageJ.

3. Calculate the ratio of epithelial tree size to whole mount size, to indicate the MG formation efficiency.

\section{Data analysis}

The mammary gland formation efficiency can be assessed by the branching extent of the mammary epithelial tree. The pictures of stained whole mount could be analyzed by ImageJ. Gate the area of epithelial tree and calculate the size as A (Figure 2); then gate the area of the whole mount and calculate the size as $B$. Then the MG formation efficiency can be quantified by the ratio of $A / B$, which ranges from 0 to 1 ( 0 means no $M G$ formation and 1 means the entire reconstruction of MG). Figure 2 shows three examples of reconstructed MG with different efficiencies. 

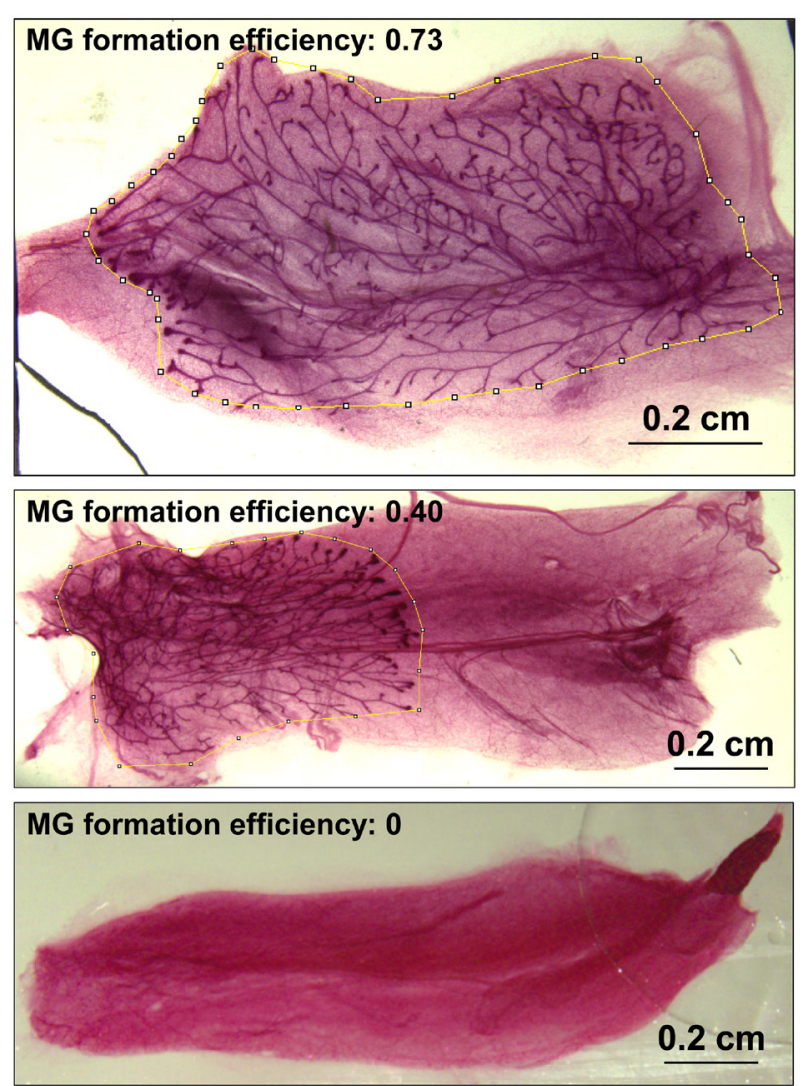

Figure 2. Examples of reconstructed mammary gland. MG formation efficiency was calculated as the ratio of epithelial tree size to whole mount size. The area of the epithelial tree was indicated as yellow circle. The top 2 MG were reconstructed from implantation of 2,000 basal cells, and the bottom MG showed no epithelial tree formation after implantation of 2,000 luminal cells. The recipient mice were kept for 8 weeks after the implantation.

\section{$\underline{\text { Notes }}$}

1. The age and body weight of the recipient mice are very important for the experiment. Select 3week-old virgin female mice with body weight less than $12 \mathrm{~g}$ to ensure that mammary ductal growth has not been initiated.

2. The implantation buffer and the cell type could greatly affect the MG formation efficiency. We found that adding matrigel (30\%-50\%) to replace FBS could improve the MG formation efficiency.

3. Adjust the MG cell concentration according to your requirement, as only about $10-20 \mu \mathrm{l}$ of cells can be implanted in the cleared fat pad. For example, if 2,000 cells will be implanted, make the cell concentration as $2 \times 10^{5}$ cells / $\mathrm{ml}$ in implantation buffer, and inject $10 \mu \mathrm{l}$ cells per pad.

4. Stain the MG with carmine-alum staining solution at $4{ }^{\circ} \mathrm{C}$ overnight to several days, depending on the thickness of MG. Staining could be terminated when the red dye penetrated whole mount. 


\section{Recipes}

1. Implantation buffer $(1 \mathrm{ml})$

$0.6 \mathrm{ml} \mathrm{DMEM} / \mathrm{F} 12$ medium

$0.3 \mathrm{ml}$ FBS

$0.1 \mathrm{ml} 0.4 \%$ trypan blue

The trypan blue was used to indicate whether the cells were successfully injected as the volume was quite limited

2. Avertin $(200 \mathrm{ml})$

a. Dissolve $2.5 \mathrm{~g} \mathrm{2,2,2-Tribromoethanol} \mathrm{with} 5 \mathrm{ml}$ 2-Methyl-2-butanol by using a magnetic stirrer in the dark overnight

b. Then add $195 \mathrm{ml}$ PBS and mix well. Filter the mixture through a $0.2 \mu \mathrm{m}$ Nalgene syringe filter

c. The Avertin could be kept in the dark at $4{ }^{\circ} \mathrm{C}$ for several months

3. Carnoy's fix buffer $(500 \mathrm{ml})$

$300 \mathrm{ml}$ ethanol

$150 \mathrm{ml}$ chloroform

$50 \mathrm{ml}$ acetic acid

4. Carmine-alum staining solution $(500 \mathrm{ml})$

a. Add $1 \mathrm{~g}$ carmine and $2.5 \mathrm{~g}$ aluminum potassium sulfate to $500 \mathrm{ml}$ demineralized $\mathrm{H}_{2} \mathrm{O}$

b. Boil the mixture and let it cool down to room temperature before use

c. The carmine-alum staining solution could be kept at room temperature and re-used up to three times

\section{Acknowledgments}

This protocol was adapted from our previous work (Sun et al., 2018), which was supported by Chair Professor Grant CPG 2017-00016-FHS and Startup Research Grant SRG 2017-00045-FHS, by University of Macau, Macau SAR, China, Macao Science and Technology Development Fund Grants 065/2015/A2 and 094/2015/A3, and by National Natural Science Foundation of China Grant 81602587.

\section{Competing interests}

The authors declare that they have no conflict of interest.

\section{Ethics}

This protocol involving mice has been approved by the University of Macau Animal Ethics 
Committee under protocol UMAEC-050-2015.

\section{References}

1. Hennighausen, L. and Robinson, G. W. (2005). Information networks in the mammary gland. Nat Rev Mol Cell Biol 6(9): 715-725.

2. Prater, M. D., Petit, V., Alasdair Russell, I., Giraddi, R. R., Shehata, M., Menon, S., Schulte, R., Kalajzic, I., Rath, N., Olson, M. F., Metzger, D., Faraldo, M. M., Deugnier, M. A., Glukhova, M. A. and Stingl, J. (2014). Mammary stem cells have myoepithelial cell properties. Nat Cell Biol 16(10): 942-950, 941-947.

3. Sun, H., Xu, X. and Deng, C. (2020). Preparation of Single Epithelial Cells Suspension from Mouse Mammary Glands. Bio-protocol 10(4): e3530.

4. Sun, H., Miao, Z., Zhang, X., Chan, U. I., Su, S. M., Guo, S., Wong, C. K. H., Xu, X. and Deng, C. X. (2018). Single-cell RNA-Seq reveals cell heterogeneity and hierarchy within mouse mammary epithelia. J Biol Chem 293(22): 8315-8329.

5. Van Keymeulen, A., Rocha, A. S., Ousset, M., Beck, B., Bouvencourt, G., Rock, J., Sharma, N., Dekoninck, S. and Blanpain, C. (2011). Distinct stem cells contribute to mammary gland development and maintenance. Nature 479(7372): 189-193.

6. Wang, D., Cai, C., Dong, X., Yu, Q. C., Zhang, X. O., Yang, L. and Zeng, Y. A. (2015). Identification of multipotent mammary stem cells by protein $\mathrm{C}$ receptor expression. Nature 517(7532): 81-84. 